Bull. Korean Math. Soc. 49 (2012), No. 2, pp. 307-317

http://dx.doi.org/10.4134/BKMS.2012.49.2.307

\title{
EQUIDISTRIBUTION OF PERIODIC POINTS OF SOME AUTOMORPHISMS ON K3 SURFACES
}

\author{
Chong Gyu Lee
}

\begin{abstract}
We say $\left(W,\left\{\phi_{1}, \ldots, \phi_{t}\right\}\right)$ is a polarizable dynamical system of several morphisms if $\phi_{i}$ are endomorphisms on a projective variety $W$ such that $\otimes \phi_{i}^{*} L$ is linearly equivalent to $L^{\otimes q}$ for some ample line bundle $L$ on $W$ and for some $q>t$. If $q$ is a rational number, then we have the equidistribution of small points of given dynamical system because of Yuan's work [13]. As its application, we can build a polarizable dynamical system of an automorphism and its inverse on a $K 3$ surface and can show that its periodic points are equidistributed.
\end{abstract}

\section{Introduction}

The study of algebraic dynamics blooms after Northcott proved the arithmetic property of a dynamical system of a morphism on a projective space. Szpiro, Ullmo and Zhang [12] started one direction of algebraic dynamics, the equidistribution of small points. After various research of Bilu [3] on some variety with group structure and of Baker and Rumely [2], Chambert-loir [4], and Favre and Rivera-Letelier [7] on the dynamical equidistribution on $\mathbb{P}^{1}$, Yuan [13] proved the general equidistribution theorem: let $\phi$ be a polarizable endomorphism. Then, we have an ample line bundle $L$ with a semipositive dynamical metric $\|\cdot\|_{\phi}$ defined by Zhang [14], then we have the equidistribution of small points with respect to the height function corresponding to $\bar{L}=\left(L,\|\cdot\|_{\phi}\right)$.

For the dynamical equidistribution, the "polarizability" condition is very important because it guarantees that we can define a sequence of metrics defined on the same line bundle. If $\phi$ is not polarizable, then, metrics $\phi^{k^{*}}\|\cdot\| \frac{1}{q^{k}}$ may be defined on different line bundles for each $k$ so that "convergence" of given sequence of metrics doesn't make sense.

Still, we have hope because of Kawaguchi's idea. He [8] suggested the polarizable dynamical system of several morphisms:

Received October 31, 2010; Revised March 8, 2011.

2010 Mathematics Subject Classification. Primary 14G40, 11G50; Secondary 37P30, $14 \mathrm{~J} 28$.

Key words and phrases. equidistribution, height, dynamical system, $K 3$ surface, automorphism. 
Definition 1.1. Let $W$ be a projective variety, let $L$ be an ample line bundle on $W$ and let $M=\left\{\phi_{1}, \ldots, \phi_{t}: W \rightarrow W\right\}$ be a finite set of endomorphisms on $W$. We say that a dynamical system of several morphisms $(W, M)$ is polarizable if

$$
\bigotimes_{i=1}^{t} \phi_{i}^{*} L \sim L^{\otimes q}
$$

for some rational number $q>t$.

His idea makes a way to study the dynamics of some automorphisms. In general, an automorphisms on a projective variety is not polarizable in general. For example, if the rank of Néron-Severi Group of $W$ is one, there is no polarizable automorphism. However, we have a good counter part, the inverse map. The existence of the inverse map makes a dynamical system of an automorphism better; a dynamical system of several morphisms $\left(W, M=\left\{\phi_{1}, \ldots, \phi_{t}\right\}\right)$ actually works with the monoid generated by $M$. If $M$ consists of an automorphism $\sigma$ and its inverse, the monoid $\mathcal{M}$ generated by $M$ is exactly $\left\{\sigma^{k} \mid k \in \mathbb{Z}\right\}$ and hence $\mathcal{M}$-preperiodic point is essentially ' $\sigma$-preperiodic or $\sigma^{-1}$-prepriodic points'. And, all preperiodic points of automorphism $\sigma^{k}$ is actually periodic, and $\sigma^{k}$ and $\sigma^{-k}$ share that same set of periodic points for all $k$. Thus, to study the properties of periodic points of $\sigma$, we can use a dynamical system $\left(W,\left\{\sigma, \sigma^{-1}\right\}\right)$ instead of $(W, \sigma)$. In Section 2, we have examples of polarizable dynamical systems of an automorphism and its inverse on $K 3$ surfaces.

The main purpose of this paper is to show that we have the dynamical equidistribution for dynamical systems of several morphisms and to apply this result on some automorphisms on $K 3$ surface. In Section 3, we will combine Kawaguchi's and Yuan's results to prove the equidistribution of small points:

Theorem 1.2. Let $W$ be a projective variety of dimension $n$ over a number field $K$ and let $M=\left\{\phi_{1}, \ldots, \phi_{t}\right\}$ be a finite set of endomorphisms on $W$. Suppose that $(W, M)$ is a polarizable with respect to $\mathcal{L}$ and $\left\{x_{m}\right\}$ be a generic and small sequence. Then, the a sequence of probability measure on the Galois orbit of $x_{m}$ weakly converges to the dynamical measure at every place $v$ :

$$
\frac{1}{\operatorname{deg} x_{m}} \sum_{y \in \Gamma_{x_{m}}} \delta_{y} \rightarrow \mu_{M, v}
$$

where $\Gamma_{x_{m}}$ is the Galois orbit or $x_{m}$ and $\mu_{M, v}=\frac{c_{1}(\mathcal{L})_{v}^{n}}{\operatorname{deg}_{L} W}$ is the dynamical probability measure of the dynamical system $(W, M)$ on the analytic space $W_{K_{v}}^{a n}$.

In Section 5, we will show that we can find a generic and small sequence of periodic points. Thus, we can find some properties of the set of periodic points of some automorphisms on $K 3$ surfaces.

Theorem 1.3. Let $W$ be a projective variety defined over a number field $K$ and let $M=\left\{\sigma, \sigma^{-1}\right\}$ be a set of an automorphism and its inverse on $W$. Suppose that $(W, M)$ is polarizable. Then, $\operatorname{Per}(\sigma)$ is Zariski dense. 
Acknowledgements. The author would like to thank Xinyi Yuan for helpful discussions and comments for paper, thank Joseph H. Silverman for suggesting ideal for the proof of Theorem 4. Also thanks to Shu Kawaguchi and Jordan Ellenberg for useful comments.

\section{Polarizable dynamical systems of automorphisms on $K 3$ surfaces}

We have lots of interesting examples of polarizable dynamical systems of several morphisms on $K 3$ surfaces. We refer $[5,6,10,11]$ for general reference for intersection Theory and height functions.

\subsection{K3 surface with two involutions, I}

The space of $K 3$ surfaces is a 19-dimensional object up to isomorphism. And, a family $\mathcal{F}$ of $K 3$ surfaces in $\mathbb{P}^{2} \times \mathbb{P}^{2}$ defined by an intersection of hypersurfaces of bidegree $(1,1)$ and $(2,2)$ is a 18-parameter family of isomorphism classes of nonsingular surfaces. For details of such $K 3$ surfaces, we refer $[9, \S 7.4]$. When $S$ is defined in $\mathbb{P}^{2} \times \mathbb{P}^{2}$, the we have restrictions of projections $\pi_{i}: P_{1}^{1} \times P_{2}^{1} \rightarrow \mathbb{P}_{i}^{1}$ on $S$. Then, because of the definition of $S,\left.\pi_{i}\right|_{S}$ are generically two-to-one maps. So, we can define rational maps $\iota_{i}: S \rightarrow S$ such that

$$
\iota_{i}(P):=Q \text { if } \pi_{i}^{-1}\left(\pi_{i}(P)\right)=\{P, Q\} .
$$

Then, we can check that $\iota_{1}$ and $\iota_{2}$ are automorphisms of order 2 for all surfaces in $\mathcal{F}$ except a Zariski closed subset of $\mathcal{F}$. Since $\iota_{i}$ are morphisms of finite order, their dynamical property is not so interesting. However, $\iota_{1}$ and $\iota_{2}$ are not commutative so that they will generate a 'dynamically interesting' automorphism.

Example 2.1. Let $S \subset \mathbb{P}^{2} \times \mathbb{P}^{2}$ be a $K 3$ surface defined by an intersection of hypersurfaces of bidegree $(1,1)$ and $(2,2)$ with two involutions $\iota_{1}, \iota_{2}$, let $\pi_{i}$ be the projection map onto $i$-th component and let $L_{i}=\pi_{i}^{*} \mathcal{O}_{\mathbb{P}^{2}}(1)$. Then, we have

and hence

$$
\iota_{i}^{*} L_{i}=L_{i}, \quad \iota_{i}^{*} L_{j}=L_{i}^{\otimes 4} \otimes L_{j}^{\otimes-1}
$$

$$
\iota_{1}^{*} L \otimes \iota_{2}^{*} L=L^{\otimes 4},
$$

where $L=L_{1} \otimes L_{2}$ is an ample line bundle. Therefore, $\left(S,\left\{\iota_{1}, \iota_{2}\right\}\right)$ is a polarizable dynamical system.

Example 2.2. Let $S$ be the $K 3$ surface defined on Example 2.1. Define $\sigma_{1}=$ $\iota_{2} \circ \iota_{1}$ and $\sigma_{2}=\iota_{1} \circ \iota_{2}=\sigma_{1}^{-1}$. Then, $\left(S,\left\{\sigma_{1}, \sigma_{2}\right\}\right)$ is a polarizable dynamical system: we have

$$
\sigma_{i}^{*} L_{i}=L_{i}^{\otimes-1} \otimes L_{j}^{\otimes 4}, \quad \sigma_{i}^{*} L_{j}=L_{i}^{\otimes-4} \otimes L_{j}^{\otimes 15}
$$

and

$$
\sigma_{1}^{*} L \otimes \sigma_{2}^{*} L=L^{\otimes 14} .
$$




\subsection{K3 surface with two involutions, II}

There is another way of defining $K 3$ surface in $\mathbb{P}^{2} \times \mathbb{P}^{2}$, intersecting two hypersurfaces of bidegree $(1,2)$ and $(2,1)$. With the same method on $[9, \S 7.4]$, we can calculate the dimension of such family; the number of parameters of defining equations

$$
\sum_{0 \geq i \geq j \geq 2} \sum_{0 \geq k \geq 2} A_{i j k} x_{i} x_{j} y_{k} \text { and } \sum_{0 \geq l \geq 2} \sum_{0 \geq m \geq n \geq 2} B_{m n l} x_{l} y_{m} y_{n}
$$

is $(18-1)+(18-1)$ and the dimension of $\mathrm{PGL}_{3}$, the isometry group of each $\mathbb{P}^{2}$, is 8 . Hence the dimension of the family of such $K 3$ surface is $17+17-8-8=18$ again. Since $S$ is still defined in $\mathbb{P}^{2} \times \mathbb{P}^{2}, S$ has two involutions from projection maps.

Example 2.3. Let $S \subset \mathbb{P}^{2} \times \mathbb{P}^{2}$ be a $K 3$ surface generated by intersecting two hypersurfaces of bidegree $(1,2)$ and $(2,1)$ with two involutions $\iota_{1}, \iota_{2}$. Let $\pi_{i}$ be the projection map onto $i$-th component and $L_{i}=\pi_{i}^{*} \mathcal{O}_{\mathbb{P}^{2}}(1)$. Then, we have

$$
\iota_{i}^{*} L_{i}=L_{i}, \quad \iota_{i}^{*} L_{j}=L_{i}^{\otimes-1} \otimes L_{j}^{\otimes 5} .
$$

Since $L=L_{1} \otimes L_{2}$ is ample, we get

$$
\iota_{1}^{*} L \otimes \iota_{2}^{*} L=L^{\otimes 5}
$$

and hence get a polarizable dynamical system.

Example 2.4. Let $S$ be the $K 3$ surface defined on Example 2.3. Define $\sigma_{1}=$ $\iota_{2} \circ \iota_{1}$ and $\sigma_{2}=\iota_{1} \circ \iota_{2}=\sigma_{1}^{-1}$. Then, $\left(S,\left\{\sigma_{1}, \sigma_{2}\right\}\right)$ is a polarizable dynamical system: we have

$$
\sigma_{i}^{*} L_{i}=L_{i}^{\otimes-1} \otimes L_{j}^{\otimes 5}, \quad \sigma_{i}^{*} L_{j}=L_{i}^{\otimes-5} \otimes L_{j}^{\otimes 24}
$$

and hence

for all $L \in\left\langle L_{1}, L_{2}\right\rangle$.

$$
\sigma_{1}^{*} L \otimes \sigma_{2}^{*} L=L^{\otimes 23}
$$

\subsection{K3 surface with three involutions}

If we define a $K 3$ surface $S$ in $\mathbb{P}^{1} \times \mathbb{P}^{1} \times \mathbb{P}^{1}$, then the Picard number of $S$ is at least 3 , greater than that of $K 3$ surface in $\mathbb{P}^{2} \times \mathbb{P}^{2}$ by 1 . Thus we expect that the dimension of the family of $K 3$ surface is reduced by 1 ; the number of parameters for defining equations

$$
\sum_{0 \geq i \geq j \geq 2} \sum_{0 \geq k \geq l \geq 2} \sum_{0 \geq m \geq n \geq 2} A_{i j k l m n} x_{i} x_{j} y_{k} y_{l} z_{m} z_{n}
$$

is $27-1$ and the dimension of $\mathrm{PGL}_{2}$, the isometry group of each $\mathbb{P}^{1}$, is 3 . Hence the dimension of the family of $K 3$ such surfaces is $26-3-3-3=17$. Now, we have projection maps $\pi_{i}: \prod_{j=1}^{3} \mathbb{P}_{j}^{1} \rightarrow \prod_{j \neq i} \mathbb{P}_{j}^{1}$ which are generically two-to-one on $S$. So, we can define three involutions with the same method. 
Example 2.5. Let $S \subset \mathbb{P}^{1} \times \mathbb{P}^{1} \times \mathbb{P}^{1}$ be a $K 3$ surface, a hypersurface of bidegree $(2,2,2)$ with three involutions $\iota_{1}, \iota_{2}, \iota_{3}$. Let $\pi_{i}$ be the projection map onto $i$-th component and $L_{i}=\pi_{i}^{*} \mathcal{O}_{\mathbb{P}^{1}}(1)$. Then, we have

$$
\iota_{i}^{*} L_{j}=L_{j} \text { for } i \neq j, \quad \iota_{i}^{*} L_{i}=L_{i}^{\otimes-1} \otimes L_{j}^{\otimes 2} \otimes L_{k}^{\otimes 2} .
$$

Hence, let $L=L_{1} \otimes L_{2} \otimes L_{3}$ and get

$$
\iota_{1}^{*} L \otimes \iota_{2}^{*} L \otimes \iota_{3}^{*} L=L^{\otimes 5} .
$$

So, $\left(S,\left\{\iota_{1}, \iota_{2}, \iota_{3}\right\}\right)$ is polarizable.

Example 2.6. Let $S$ be the $K 3$ surface defined on Example 2.5. Consider $\tau_{1}=\iota_{3} \circ \iota_{2} \circ \iota_{1}, \tau_{2}=\tau_{1}^{-1}$. Then,

$$
\begin{aligned}
& \tau_{1}^{*} L_{1}=\iota_{3}^{*} \iota_{2}\left(L_{1}^{\otimes-1} \otimes L_{2}^{\otimes 2} \otimes L_{3}^{\otimes 2}\right) \\
&=\iota_{3}^{*}\left(L_{1}^{\otimes 3} \otimes L_{2}^{\otimes-2} \otimes L_{3}^{\otimes 6}\right)=L_{1}^{\otimes 15} \otimes L_{2}^{\otimes 10} \otimes L_{3}^{\otimes-6}, \\
& \tau_{1}^{*} L_{2}= \iota_{3}^{*} \iota_{2}^{*}\left(L_{2}\right)=\iota_{3}^{*}\left(L_{1}^{\otimes 2} \otimes L_{2}^{\otimes-1} \otimes L_{3}^{\otimes 2}\right)=L_{1}^{\otimes 6} \otimes L_{2}^{\otimes 3} \otimes L_{3}^{\otimes-2}, \\
& \tau_{1}^{*} L_{3}=\iota_{3}^{*} \iota_{2}^{*}\left(L_{3}\right)=\iota_{3}^{*} L_{3}=L_{1}^{\otimes 2} \otimes L_{2}^{\otimes 2} \otimes L_{3}^{\otimes-1}, \\
& \tau_{2}^{*} L_{1}=\iota_{1}^{*} \iota_{2}^{*}\left(L_{1}\right)=\iota_{1}^{*} L_{1}=L_{1}^{\otimes-1} \otimes L_{2}^{\otimes 2} \otimes L_{3}^{\otimes 2}, \\
& \tau_{2}^{*} L_{2}=\iota_{1}^{*} \iota_{2}^{*}\left(L_{2}\right)=\iota_{1}^{*}\left(L_{1}^{\otimes 2} \otimes L_{2}^{\otimes-1} \otimes L_{3}^{\otimes 2}\right)=L_{1}^{\otimes-2} \otimes L_{2}^{\otimes 3} \otimes L_{3}^{\otimes 6}, \\
& \tau_{2}^{*} L_{3}=\iota_{1}^{*} \iota_{2}\left(L_{1}^{\otimes 2} \otimes L_{2}^{\otimes 2} \otimes L_{3}^{\otimes-1}\right) \\
&=\iota_{1}^{*}\left(L_{1}^{\otimes 3} \otimes L_{2}^{\otimes-2} \otimes L_{3}^{\otimes 6}\right)=L_{1}^{\otimes-6} \otimes L_{2}^{\otimes 10} \otimes L_{3}^{\otimes 15}, \\
& {\left[\tau_{1}^{*} \otimes \tau_{2}^{*}\right]\left(L_{1}^{\otimes a} \otimes L_{2}^{\otimes b} \otimes L_{3}^{\otimes c}\right)=\left(L_{1}^{\otimes 15 a+6 b+2 c} \otimes L_{2}^{\otimes 10 a+3 b+2 c} \otimes L_{3}^{\otimes-6 a-2 b-c}\right) } \\
& \quad \otimes\left(L_{1}^{\otimes-a-2 b-6 c} \otimes L_{2}^{\otimes 2 a+3 b+10 c} \otimes L_{3}^{\otimes 2 a+6 b+15 c}\right) \\
&=L_{1}^{\otimes 14 a+4 b-4 c} \otimes L_{2}^{\otimes 12 a+6 b+12 c} \otimes L_{3}^{\otimes-4 a+4 b+14 c} .
\end{aligned}
$$

Let $L=L_{1} \otimes L_{2}^{\otimes 2} \otimes L_{3}$. Then,

$$
\tau_{1}^{*} L \otimes \tau_{2}^{*} L \sim L^{\otimes 18}
$$

and hence $\left(S,\left\{\tau_{1}, \tau_{2}\right\}\right)$ is polarizable. More precisely, let $L_{\alpha, \beta}=\left(L_{1} \otimes L_{2}\right)^{\otimes \alpha} \otimes$ $\left(L_{1}^{-1} \otimes L_{3}\right)^{\otimes \beta}$, then

$$
\tau_{1}^{*} L_{\alpha, \beta} \otimes \tau_{2}^{*} L_{\alpha, \beta} \sim L_{\alpha, \beta}^{\otimes 18}
$$

Similarly, automorphisms $\tau^{\prime}=\iota_{1} \circ \iota_{3} \circ \iota_{2}, \tau^{\prime \prime}=\iota_{2} \circ \iota_{1} \circ \iota_{3}$ with their inverses will generate polarizable dynamical systems respectively.

Example 2.7. Consider the following case; let $S$ be a $K 3$ surface defined on Example 2.5. Define $\sigma_{1}=\iota_{2} \circ \iota_{1}$ and $\sigma_{2}=\iota_{1} \circ \iota_{2}=\sigma_{1}^{-1}$. Then, $\left(S,\left\{\sigma_{1}, \sigma_{2}\right\}\right)$ is not a polarizable dynamical system:

$$
\begin{aligned}
& \sigma_{1}^{*} L_{1}=\iota_{2}^{*}\left(L_{1}^{\otimes-1} \otimes L_{2}^{\otimes 2} \otimes L_{3}^{\otimes 2}\right)=L_{1}^{\otimes 3} \otimes L_{2}^{\otimes-2} \otimes L_{3}^{\otimes 6}, \\
& \sigma_{1}^{*} L_{2}=\iota_{2}^{*}\left(L_{2}\right) \quad=L_{1}^{\otimes 2} \otimes L_{2}^{\otimes-1} \otimes L_{3}^{\otimes 2} \text {, } \\
& \sigma_{1}^{*} L_{3}=\iota_{2}^{*}\left(L_{3}\right) \quad=L_{3} \text {. }
\end{aligned}
$$


Therefore,

$$
\begin{aligned}
{\left[\sigma_{1}^{*} \otimes \sigma_{2}^{*}\right]\left(L_{1}^{\otimes a} \otimes L_{2}^{\otimes b} \otimes L_{3}^{\otimes c}\right)=} & \left(L_{1}^{\otimes 3 a+2 b} \otimes L_{2}^{\otimes-2 a-b} \otimes L_{3}^{\otimes 6 a+2 b+c}\right) \\
& \otimes\left(L_{1}^{\otimes-a-2 b} \otimes L_{2}^{\otimes 2 a+3 b} \otimes L_{3}^{\otimes 2 a+6 b+c}\right) \\
= & L_{1}^{\otimes 2 a} \otimes L_{2}^{\otimes 2 b} \otimes L_{3}^{\otimes 8 a+8 b+2 c} .
\end{aligned}
$$

Therefore, $L_{3}$ is the only combination of $L_{1}, L_{2}$ and $L_{3}$ which makes linear equivalence;

$$
\sigma_{1}^{*} L_{3} \otimes \sigma_{2}^{*} L_{3} \sim L_{3}^{\otimes 2}
$$

and hence $\left(S,\left\{\sigma_{1}, \sigma_{2}\right\}\right)$ is not a polarizable dynamical system in Kawaguchi's sense. Similarly, $\left(S,\left\{\iota_{1} \circ \iota_{3}, \iota_{3} \circ \iota_{1}\right\}\right),\left(S,\left\{\iota_{2} \circ \iota_{3}, \iota_{3} \circ \iota_{2}\right\}\right)$ are not polarizable.

\subsection{K3 surface in $\mathbb{P}^{1} \times \mathbb{P}^{1} \times \mathbb{P}^{1}$ with four involutions}

We have some special case. If $S$ has a fiber structure such that all fiber is isomorphic to an elliptic curve, then we can define another involution from group structure: $\iota_{4}(P)=[-1]_{E} P$ where $E$ is an elliptic fiber containing $P$ and $[-1]_{E}$ is the additive inverse map on $E$. For details, refer [1].

Example 2.8. Let $S=\mathbb{P}^{1} \times \mathbb{P}^{1} \times \mathbb{P}^{1}$ be a $K 3$ surface, a hypersurface of bidegree $(2,2,2)$ of the Picard number 4 with four involutions; $\operatorname{Pic}(S)=\left\langle L_{1}, L_{2}, L_{3}, L_{4}\right\rangle$ where $L_{4}$ corresponds to -2 -curve class containing $(x, 0,0)$. Define an automorphisms $\tau=\iota_{1} \circ \iota_{2} \circ \iota_{4}$ and $\tau^{-1}=\iota_{4} \circ \iota_{2} \circ \iota_{1}$. Then,

$$
\iota_{4}^{*} L_{1}=L_{1}, \iota_{4}^{*} L_{4}=L_{4}, \quad \iota_{4}^{*} L_{j}=L_{1}^{\otimes 8} \otimes L_{i}^{-1} \otimes L_{4} .
$$

Therefore,

$$
\tau^{*} L \otimes \tau^{-1^{*}} L=L^{\otimes 30}
$$

if

$$
L=\left(L_{1}^{\otimes 4} \otimes L_{2}^{\otimes 4} \otimes L_{3}^{\otimes 3}\right)^{\otimes \alpha} \otimes\left(L_{1}^{\otimes 8} \otimes L_{2}^{\otimes 2} \otimes L_{4}^{\otimes 3}\right)^{\otimes \beta}
$$

for some $\alpha, \beta$. Similarly, $\tau^{\prime}=\iota_{1} \circ \iota_{3} \circ \iota_{4}$ with its inverse will generate a polarizable dynamical system. But, $\zeta=\iota_{2} \circ \iota_{3} \circ \iota_{4}$ or $\zeta^{\prime}=\iota_{3} \circ \iota_{2} \circ \iota_{4}$ are polarized by $q=2$ or $q=-2$. Also, $\eta=\iota_{1} \circ \iota_{4} \circ \iota_{2}, \eta^{\prime}=\iota_{1} \circ \iota_{4} \circ \iota_{2}$ are not polarized by any ample line bundles.

\section{Dynamical equidistribution on polarizable dynamical systems of several morphisms}

The equidistribution of small points for polarizable dynamical systems of several morphisms is almost proved. Kawaguchi proved that the dynamical system of several morphisms generates the dynamical adelic metric which is semipositive. Thus, the equidistribution of small points of a polarizable dynamical system is an easy consequence of Yuan's results. In this section, we will briefly check Kawaguchi's and Yuan's results to confirm the equidistribution theory for dynamical system of several morphisms. 
Definition 3.1. Let $W$ be a projective variety defined over a number field $K$, let $\bar{L}=(L,\|\cdot\|)$ be a metrized line bundle on $W$ such that $L$ is an ample line bundle $L$ with a semipositive adelic metric $\|\cdot\|$. Then we define a height of subvarieties corresponding to $\bar{L}$ to be

$$
h_{\bar{L}}(Y):=\frac{c_{1}(\bar{L})^{d+1}}{(d+1) \operatorname{ord}_{\bar{L}} Y},
$$

where $Y$ is a subvariety of $W$ of dimension $d$ and $c_{1}$ is the curvature form.

Definition 3.2. Let $W$ be a projective variety, let $\bar{L}$ be a metrized line bundle and let $h_{\bar{L}}$ be the height function for closed subvarieties of $W$ corresponding to $\bar{L}$ and let $\left\{x_{m}\right\}$ is a sequence of points on $W$. We say $\left\{x_{m}\right\}$ is generic if any infinite subsequence of $\left\{x_{m}\right\}$ is not contained in a closed subvariety. We say $\left\{x_{m}\right\}$ is small if $h_{\bar{L}}\left(x_{m}\right)$ converges to $h_{\bar{L}}(W)$.

Theorem 3.3 ([13, Theorem 3.2]). Let $W$ be a projective variety of dimension $n$ over a number field $K$, and let $\bar{L}$ be a metrized line bundle over $W$ with semipositive adelic metric. Suppose that $\left\{x_{m}\right\}$ is an infinite sequence of closed points on $W$ which is generic and small with respect to $h_{\bar{L}}$. Then, for any place $v$ of $K$, the Galois orbit of sequence $\left\{x_{m}\right\}$ are equidistributed in the analytic space $W_{K_{v}}^{a n}$ with respect to the canonical measure $d \mu_{v}=c_{1}(\bar{L})_{v}^{n} / \operatorname{deg}_{\bar{L}} W$ :

$$
\frac{1}{\operatorname{deg} x_{m}} \sum_{y \in \Gamma x_{m}} \delta_{y} \text { weakly converges to } d \mu_{v} \text {. }
$$

Remark 3.4. In Theorem 3.3, we should assume that $L$ is $\mathbb{Q}$-divisor. Actually we will use the integral model $(\mathcal{W}, \mathcal{L})$ of $\left(W, L^{\otimes e}\right)$ where $\mathcal{L}$ on the generic fiber $\mathcal{W}_{\mathbb{Q}}$ is an integral line bundle $L^{\otimes e}$. Thus, $L \in \operatorname{Pic}(W) \otimes \mathbb{Q}$. Using $\mathcal{L}$ instead of $L$ implies this fact.

Theorem 3.5 ([8, Theorems A and B]). Let $W$ be a projective variety defined over a number field $K$, let $L$ be an ample line bundle on $W$ and let $M=\left\{\phi_{1}, \ldots, \phi_{t}\right\}$ be a set of endomorphisms on $W$. Suppose that $(W, M)$ is polarizable with respect to $L$ :

$$
\bigotimes_{i=1}^{t} \phi_{i}^{*} L=L^{\otimes q}
$$

where $q>t$. Then,

(1) There is a unique continuous metric $\|\cdot\|_{M}$, called an admissible metric on $L$ with $\|\cdot\|_{M}^{q}=\tau^{*}\left(\phi_{1}^{*}\|\cdot\|_{M}^{q} \cdots \phi_{t}^{*}\|\cdot\|_{M}^{q}\right)$ where $\tau: L^{\otimes q} \rightarrow \bigotimes \phi_{i}^{*} L$ is an isomorphism.

(2) Let $\bar{L}=\left(L,\|\cdot\|_{M}\right)$ be the line bundle with an admissible metric. Then, there exists a unique real-valued function

$$
\widehat{h}_{\bar{L}}: W(\bar{K}) \rightarrow \mathbb{R}
$$

with the following properties: 
(a) $\widehat{h}_{\bar{L}}$ is a Weil height function corresponding to $L$.

(b) $\sum_{i=1}^{k} \widehat{h}_{\bar{L}}\left(\phi_{i}(x)\right)=q \cdot \widehat{h}_{\bar{L}}(x)$ for all $x \in W(\bar{K})$.

(3) $\widehat{h}_{\bar{L}} \geq 0$ for all $x \in W(\bar{K})$.

Remark 3.6. The condition $q>t$ is necessary because the number of $N$ combinations of $\phi_{i}$ 's in $M$ is $t^{N}$ while the growth rate of height is $q^{N}$. More precisely, the canonical height function, if exists, is of the form

$$
h_{\bar{L}}(P)=\lim _{N \rightarrow \infty} \frac{1}{q^{N}} \sum_{F \in \mathcal{M}_{N}} h_{L}(F(P)) .
$$

Therefore, if $q \leq t$, then it may not shrink at prepriodic points. For example, if $q=t$ and $P$ is a common fixed point of $M$ such that $h_{L}(P) \neq 0$, then

$$
h_{\bar{L}}(P)=\lim _{N \rightarrow \infty} \frac{1}{q^{N}} \sum_{F \in \mathcal{M}_{N}} h_{L}(F(P))=h_{L}(P)
$$

so that $P$ may not be a root of the canonical height function. Thus, even we can build a semipositive metric, it is not compatible with the original dynamical system.

Now, combining previous two lemmas, we get equidistribution of small points for the polarizable dynamical system of several morphisms:

Theorem 1.2. Let $W$ be a projective variety of dimension $n$ defined over a number field $K$ and let $M=\left\{\phi_{1}, \ldots, \phi_{t}\right\}$ be a finite set of endomorphisms on $W$. Suppose that $(W, M)$ is a polarizable with respect to $\mathcal{L}$ and $\left\{x_{m}\right\}$ be a generic and small sequence. Then, the a sequence of probability measure on the Galois orbit of $x_{m}$ weakly converges to the dynamical measure at every place $v$ :

$$
\frac{1}{\operatorname{deg} x_{m}} \sum_{y \in \Gamma_{x_{m}}} \delta_{y} \rightarrow \mu_{M, v},
$$

where $\Gamma_{x_{m}}$ is the Galois orbit of $x_{m}$ and $\mu_{M, v}=\frac{c_{1}(\mathcal{L})_{v}^{n}}{\operatorname{deg}_{L} W}$ is the probability $M$-invariant measure on the analytic space $W_{K_{v}}^{a n}$.

Proof. Theorem 3.5 says that we have the dynamical adelic metric on $\mathcal{L}$. Such metric is semipositive because it is defined by the limit of positive metrics. Hence, we can apply Theorem 3.3, to get the desired result.

\section{Periodic points of automorphisms}

In previous section, we have the equidistribution of small points for polarizable dynamical systems of several morphisms. To show that periodic points are equidistributed, we need to show that there is a generic sequence of periodic points and $h_{\bar{L}}(W)=0$. In this section, we will prove that the set of periodic points is Zariski dense in $W$ which will complete the equidistribution of periodic points. 
Lemma 4.1. Let $\sigma: W \rightarrow W$ be an automorphism on a projective variety $W$ such that $\left(W,\left\{\sigma, \sigma^{-1}\right\}\right)$ is polarizable. Then, $\left(W,\left\{\sigma^{m}, \sigma^{-m}\right\}\right)$ is also polarizable for all $m \in \mathbb{Z}$.

Proof. Let

$$
\sigma^{*} L \otimes \sigma^{-1^{*}} L=L^{\otimes q} \quad \text { where } q>2
$$

Then,

$$
\sigma^{2^{*}} L \otimes \sigma^{-2^{*}} L=L^{\otimes q^{2}-2}
$$

Suppose

$$
L_{m}=\sigma^{l^{*}} m \otimes \sigma^{-l^{*}} L=L^{\otimes q_{m}} \quad \text { where } q_{m}>2
$$

and $q_{m}-q_{m-1}>2$ holds for $m=m_{0}-1, m_{0}$. Then,

$$
\sigma^{*} L_{m_{0}} \otimes \sigma^{-1^{*}} L_{m_{0}}=L^{\otimes q \cdot q_{m_{0}}} .
$$

On the other hand,

$$
\sigma^{*} L_{m_{0}} \otimes \sigma^{-1^{*}} L_{m_{0}}=\sigma^{m_{0}+1^{*}} L \otimes \sigma^{m_{0}-1^{*}} L \otimes \sigma^{-m_{0}+1^{*}} L \otimes \sigma^{-m_{0}-1^{*}} L
$$

and hence

$$
\sigma^{m_{0}+1^{*}} L \otimes \sigma^{-m_{0}-1^{*}} L=L^{\otimes q \cdot q_{m_{0}}-q_{m_{0}-1}} .
$$

Therefore,

$$
\sigma^{m_{0}+1^{*}} L \otimes \sigma^{-m_{0}-1^{*}} L=L^{\otimes q_{m_{0}}},
$$

where $q_{m_{0}}=q \cdot q_{m_{0}+1}-q_{m_{0}} \geq 2 q_{m_{0}}-q_{m_{0}-1}>2$. Moreover, $q_{m_{0}+1}-q_{m_{0}}=$ $(q-1) q_{m_{0}}-q_{m_{0}-1}>q_{m_{0}}-q_{m_{0}-1}>2$. Therefore, $\left(W,\left\{\sigma^{m}, \sigma^{-m}\right\}\right)$ is also polarizable for all $m \in \mathbb{Z}$ by induction.

Theorem 1.3. Let $W$ be a projective variety defined over a number field $K$ and let $M=\left\{\phi, \phi^{-1}\right\}$ be an automorphism and its inverse on $W$. Suppose that $(W, M)$ is polarizable with some integer $q>2$. Then, $\operatorname{Per}(\phi)$ is Zariski dense.

Proof. Suppose that the Zariski closure of $\mathcal{S}$ is $\mathbf{C}$ which has finitely many irreducible components $C_{1}, \ldots, C_{r}$ of codimension $l_{i}$. Choose a $C_{i_{0}}$ which has the lease codimension $l_{i_{0}}$. Then, $\sigma\left(C_{i_{0}}\right)$ is still an irreducible component of $\mathbf{C}$. Since there are only finitely many irreducible components, there is a component $C$ of codimension $l$ fixed by $\sigma^{m}$ for some $m>0$. Furthermore, since $\sigma$ is an automorphism, $\sigma^{*}$ is an automorphism on function fields $K\left(C_{1}\right)$. Thus, $\sigma_{*}^{m} C=C$. Similarly, $\sigma_{*}^{-m} C=C$. Let $(L)^{l}=L \cdots L$ be $l$-time self intersection of $L$. Then, by projection formula for intersection, we have

$(L)^{l} \cdot C=(L)^{l} \cdot \sigma_{*}^{m} C=\left(\sigma^{m *} L\right)^{l} \cdot C \quad$ and $(L)^{l} \cdot C=(L)^{l} \cdot \sigma_{*}^{m} C=\sigma^{m *} L \cdot C$.

Combining them to get

$$
\left(L^{\otimes 2}\right)^{l} \cdot C=\sigma^{m *} L \cdot C+\sigma^{m *} L \cdot C_{1}=\left(\sigma^{m *} L \otimes \sigma^{m *} L\right) \cdot C .
$$

On the other hand, by Lemma $4.1,\left(W,\left\{\sigma^{m}, \sigma^{-m}\right\}\right)$ is also polarizable. Thus,

$$
\left(L^{\otimes 2}\right)^{l} \cdot C=\left(L^{\otimes q_{m}}\right)^{l} \cdot C \quad \text { where } q_{m}>2
$$

and hence $(L)^{l} \cdot C=0$, which contradicts to the assumption that $L$ is ample. 
Corollary 4.2. Let $\sigma: W \rightarrow W$ be an automorphism on a projective variety $W$ such that $\left(W,\left\{\sigma, \sigma^{-1}\right\}\right)$ is polarizable with respect to an ample line bundle $L$. Then, a generic sequence $\left\{x_{m}\right\} \subset \operatorname{Per}(\sigma)$ is small.

Proof. Let $\bar{L}$ be metrizable line bundle with metric induced by $\left(W,\left\{\sigma, \sigma^{-1}\right\}\right)$. We know that $h_{\bar{L}}\left(x_{m}\right)=0$ if $x_{m} \in \operatorname{Per}(\sigma)$. Thus, we only have to show that $h_{\bar{L}}(W)=0$.

$[14$, Theorem 1.10] says that

$$
e_{1}(\bar{L}) \geq h_{\bar{L}}(W) \geq \frac{1}{n} \sum_{i=1}^{n} e_{i}(\bar{L}),
$$

where

$$
e_{i}(\bar{L})=\sup _{\substack{Y \subsetneq W \\ \text { Codim } Y=i}} \inf _{x \in W \backslash Y} \widehat{h}_{\bar{L}}(x) .
$$

By Theorem 1.3, $\operatorname{Per}(\sigma)$ is Zariski dense in $W$ and hence $e_{i}(\bar{L})=0$ for all $i=1, \ldots, n$ and hence $\widehat{h}_{\bar{L}}(W)=0$.

Now, Theorem 1.3 say that we can build a generic and small sequence of periodic points. Therefore, we can prove the equidistribution of periodic point:

Corollary 4.3. Let $W$ be a projective variety defined over a number field $K$ and let $M=\left\{\sigma, \sigma^{-1}\right\}$ be an automorphism and its inverse on $W$. Suppose that $(W, M)$ is polarizable with some integer $q>2$. Then, $\operatorname{Per}(\sigma)$ is equidistributed.

Proof. It is an easy consequence of Theorem 1.2. and Theorem 1.3: by Theorem 1.3, we can build a generic and small sequence $\left\{x_{m}\right\}$ in $\operatorname{Preper}(\sigma)$. And, the sequence of probability measure on Galois orbit of $x_{m}$ weakly converges to the dynamical measure by Theorem 1.2.

\section{References}

[1] A. Baragar and D. McKinnon, K3 surfaces, rational curves, and rational points, J. Number Theory 130 (2010), no. 7, 1470-1479.

[2] M. H. Baker and R. Rumely, Equidistribution of small points, rational dynamics, and potential theory, Ann. Inst. Fourier (Grenoble) 56 (2006), no. 3, 625-688.

[3] Y. Bilu, Limit distribution of small points on algebraic tori, Duke Math. J. 89 (1997), no. 3, 465-476.

[4] A. Chambert-Loir, Mesures et equidistribution sur les espaces de Berkovich, J. Reine Angew. Math. 595 (2006), 215-235.

[5] W. Fulton, Intersection Theory, Second edition, Springer-Verlag, Berlin, 1998.

[6] N. Fakhruddin, Questions on self maps of algebraic varieties, J. Ramanujan Math. Soc. 18 (2003), no. 2, 109-122.

[7] C. Favre and J. Rivera-Letelier, Equidistribution quantitative des points de petite hauteur sur la droite projective, Math. Ann. 335 (2006), no. 2, 311-361.

[8] S. Kawaguchi, Canonical heights, invariant currents, and dynamical eigensystems of morphisms for line bundles, J. Reine Angew. Math. 597 (2006), 135-173.

[9] J. H. Silverman, The Arithmetic of Dynamical System, Springer, 2007.

[10] _ Rational points on K3 surfaces: a new canonical height, Invent. Math. 105 (1991), no. 2, 347-373. 
[11] J. H. Silverman and M. Hindry, Diophantine Geometry: An introduction, Springer, 2000.

[12] L. Szpiro, E. Ullmo, and S. Zhang, Equirepartition des petits points, Invent. Math. 127 (1997), no. 2, 337-347.

[13] X. Yuan, Big line bundles over arithmetic varieties, Invent. Math. 173 (2008), no. 3, 603-649.

[14] S. Zhang, Small points and adelic metrics J. Algebraic Geom. 4 (1995), no. 2, 281-300.

Department of Mathematics

University of Illinois at Chicago

Chicago IL 60607, USA

E-mail address: phiel@math.uic.edu 